\title{
Les aménagements intérieurs de Bien-Assis
}

\section{Philippe Durin}

\section{OpenEdition}

\section{Journals}

Édition électronique

URL : http://journals.openedition.org/ccibp/418

DOI : $10.4000 /$ ccibp. 418

ISSN : 2493-7460

\section{Éditeur}

Centre international Blaise Pascal

\section{Édition imprimée}

Date de publication : 5 juillet 1983

Pagination : 10-17

ISSN : 0249-6674

\section{Référence électronique}

Philippe Durin, "Les aménagements intérieurs de Bien-Assis », Courrier du Centre international Blaise Pascal [En ligne], 5 | 1983, mis en ligne le 25 novembre 2015, consulté le 06 mai 2019. URL : http:// journals.openedition.org/ccibp/418; DOI : 10.4000/ccibp.418

Ce document a été généré automatiquement le 6 mai 2019.

Centre international Blaise Pascal 


\title{
Les aménagements intérieurs de Bien-Assis
}

\author{
Philippe Durin
}

1 Le témoignage le plus ancien de la présence de BienAssis nous est offert par le pittoresque dessin de Guillaume de Revel que l'on découvre dans son célèbre armorial (1450). 


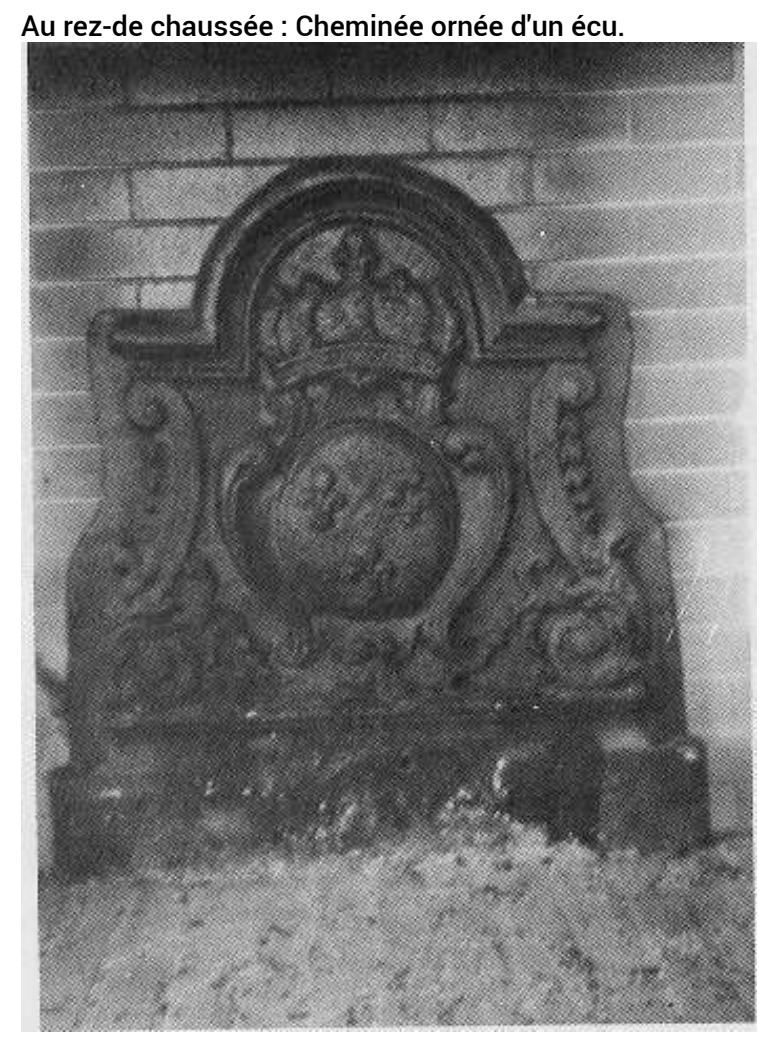

PI. 1. Cette plaque de cheminée, aux Armes de France, d'une facture tout à fait classique a protégé et décoré l'âtre d'une des cheminées monumentales de Bien-Assis. On peut penser que Blaise Pascal a profité, un jour ou l'autre, de sa bienfaisante irradiation... (Collection particulière).

Manufacture française des pneumatiques Michelin, 1912. Tous droits réservés.

2 Curieux destin celui de cet antique manoir, aux fortunes diverses, qui traversa de longues périodes obscures, avant d'entrer définitivement dans la légende, grâce aux deux fameux séjours de son illustre visiteur.

3 Solitaire, avec son propre moulin accolé à ses flancs, le château de Bien-Assis avait à l'époque pour seul voisin le couvent des Carmes Déchaussés, dont les échos de la cloche abbatiale se mêlaient à la plainte grinçante de la roue des moulins... Chantoig, Barnier, Chanteranne, clairsemés au milieu des vergers fleuris.

Dressé aux portes de la ville, on peut s'interroger sur la véritable vocation défensive, extra-muros, de ce fief rébarbatif. Farouchement entouré de double muraille, la première enceinte, sans créneau, était un simple mur. De quoi se préservait Bien-Assis? Que préservait-il ? À quelle stratégie pouvait participer ce modeste bastion dans une position défensive insolite et apparemment précaire ? Était-ce un avant-poste de la défense de la ville, ou bien le Seigneur du lieu assurait-il plus simplement sa propre sécurité ? Nul ne le saura jamais...

5 Plus d'un siècle après, on retrouve Bien-Assis sur le plan historique de la ville de Clermont, par Belleforest, daté de 1575. Les enceintes ont disparu. Seule demeure une haute cour surmontée de deux gros donjons, flanqués eux-mêmes d'un double corps de logis. Même si l'aspect purement défensif a disparu, à l'exception de deux tourelles d'angle crénelées, à l'époque où Henri III est couronné Roi de France il ne s'agit pas encore d'une aimable résidence... 
6 De la fin du XVI ${ }^{\mathrm{e}}$ siècle jusqu'à l'avènement de Louis XIV, une vaste zone d'ombre s'étend sur l'histoire de Bien-Assis. C'est cependant la période de mutation architecturale la plus importante pour le vénérable manoir qui, de forteresse, va enfin devenir une demeure de plaisance.

7 On a prêté probablement à tort à Florin Périer une vocation de bâtisseur, puisqu'une légende tenace, faussement étayée par la fameuse lettre du 5 novembre 1648 de Blaise et Jacqueline Pascal à leur sœur Gilberte, prétend que la construction des deux énormes tours carrées reliées par un corps de logis qui compose la façade sud serait due à l'initiative du beau-frère de Blaise Pascal. Non seulement cette assertion est inexacte, mais de plus, dans la lettre en question, ce n'est pas Bien-Assis qui est concerné mais la maison des Périer sise en haut de la rue du Terrail à Clermont.

8 Quoi qu'il en soit, c'est à partir de cette époque que le château, à quelques variantes près, s'est figé définitivement dans l'aspect où nous le connaissons par les documents photographiques qui ont été pris au début du siècle avant sa destruction.

9 Ensemble hétérogène, constitué d'éléments assemblés au cours des âges, Bien-Assis est le type même de la demeure auvergnate, surélevée, inachevée, où la conception originelle a été délaissée au profit d'une architecture "accommodée " aux besoins et surtout aux moyens de ses nombreux propriétaires. Même si au début du XVII ${ }^{\mathrm{e}}$ siècle le souci de sacrifier à la mode de l'époque a été l'heureux prétexte à l'édification de la grande façade sud, donnant ainsi plus d'unité et de symétrie à l'ensemble des bâtiments, on est encore loin de la grande ordonnance classique où tout est équilibre et harmonie.

10 Cependant, ce sont peut-être ces carences, ce manque de formalisme architectural, ces apports, ces retraits, ces adaptations structurelles plus ou moins heureuses, qui lui confèrent un charme indéfinissable, et qui nous font aimer cette demeure bien au-delà de son attrait historique. 
Une cheminée en anse-de-panier du XVe siècle du château de Bien-assis (Plan 51)

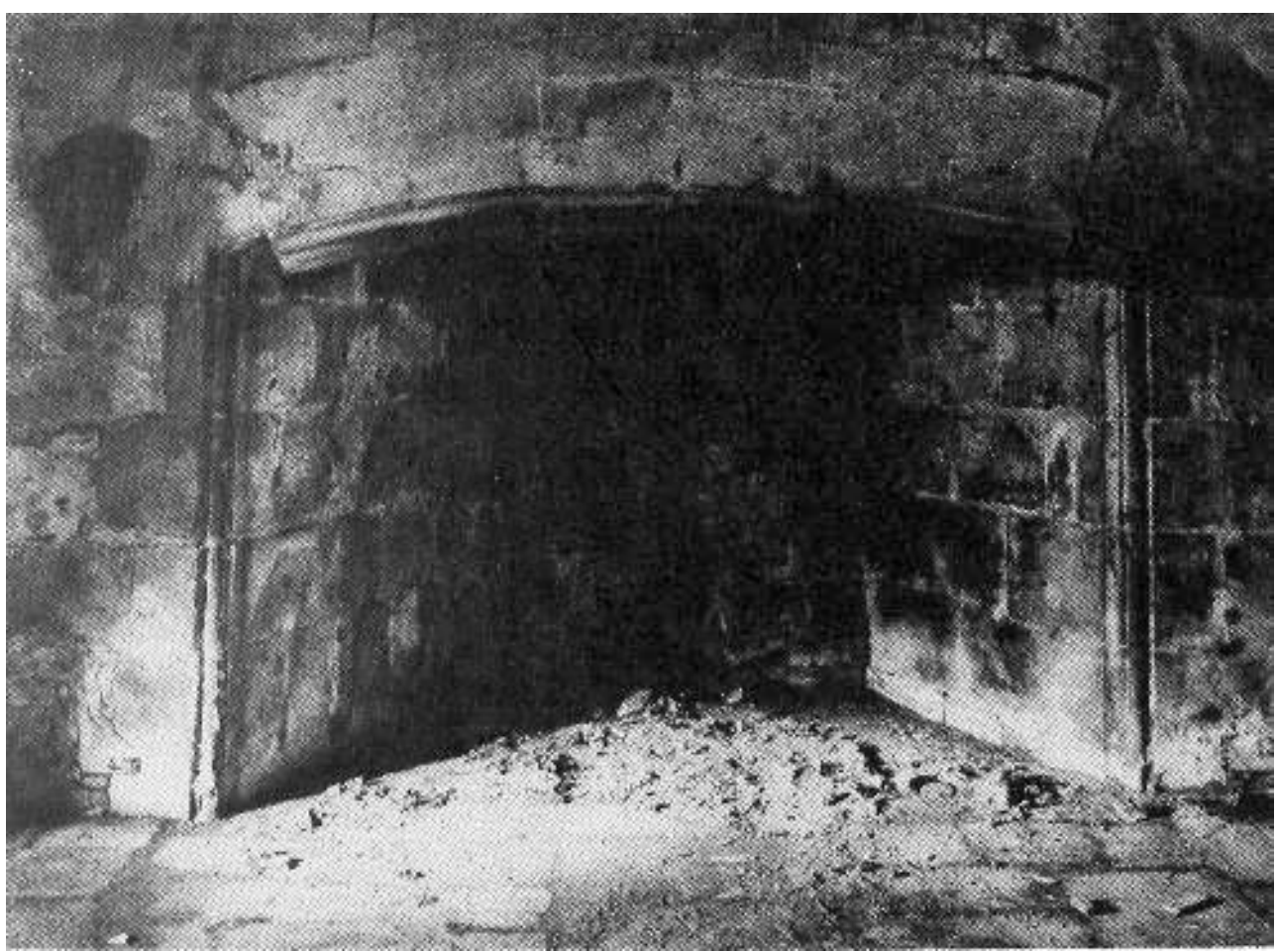

PI. 2

Manufacture française des pneumatiques Michelin, 1912. Tous droits réservés.

11 Si le passé nous a légué une iconographie intéressante sur les différents aspects extérieurs de Bien-Assis, il est en revanche beaucoup plus difficile d'appréhender ce que furent les aménagements intérieurs de la résidence d'été des Périer. Certes, les éléments architecturaux les plus intéressants ont été répertoriés et photographiés, ce qui nous a permis d'en retenir quelques-uns pour illustrer cet article, mais en fait, il existe peu de témoignages de la vie intérieure et domestique de Bien-Assis.

Fort heureusement, il nous reste le remarquable ouvrage de Christiane Marandet et L. Lafuma : «Sur les pas de Blaise Pascal - le château de Bien-Assis retrouvé » dans lequel les plans reconstitués par Maurice Marandet ainsi que ses attachants dessins situent avec beaucoup de sensibilité, mais sans complaisance, l'esprit essentiellement austère des aménagements intérieurs du château.

Fondamentalement identifiée à son terroir la vénérable bâtisse possédait toute les caractéristiques de l'architecture du pays arverne où l'apparence, le superflu, l'ornementation ostentatoire sont bannis, au profit de l'indispensable. Dans ce pays rude et laborieux, le décor, l'enjolivement, la parure sont autant d'attributs suspects parce qu'inutilement onéreux. À cet égard, on peut supposer que la famille Périer très sensible aux aspirations jansénistes, s'accommodait fort bien de la rigueur de ce cadre de vie. Il suffit de connaitre les grands escaliers sombres aux marches en lave de Volvic et aux rampes souvent indigentes, que l'on rencontre dans maints immeubles anciens, ou hôtels, de Clermont ou de Montferrand, pour imaginer que l'escalier de Bien-Assis ne devait pas déroger à cette tradition. On peut aussi penser que la même austérité était la principale caractéristique des pièces d'habitation. Mais laissons «parler » les images et reportons- 
nous à ces quelques illustrations, qui, mieux que tout discours, évoquent pour nous ce qu'étaient les aménagements intérieurs de Bien-Assis.

Évier en lave de volvic surmonté d'une niche trilobée. (Plan, 54/55).

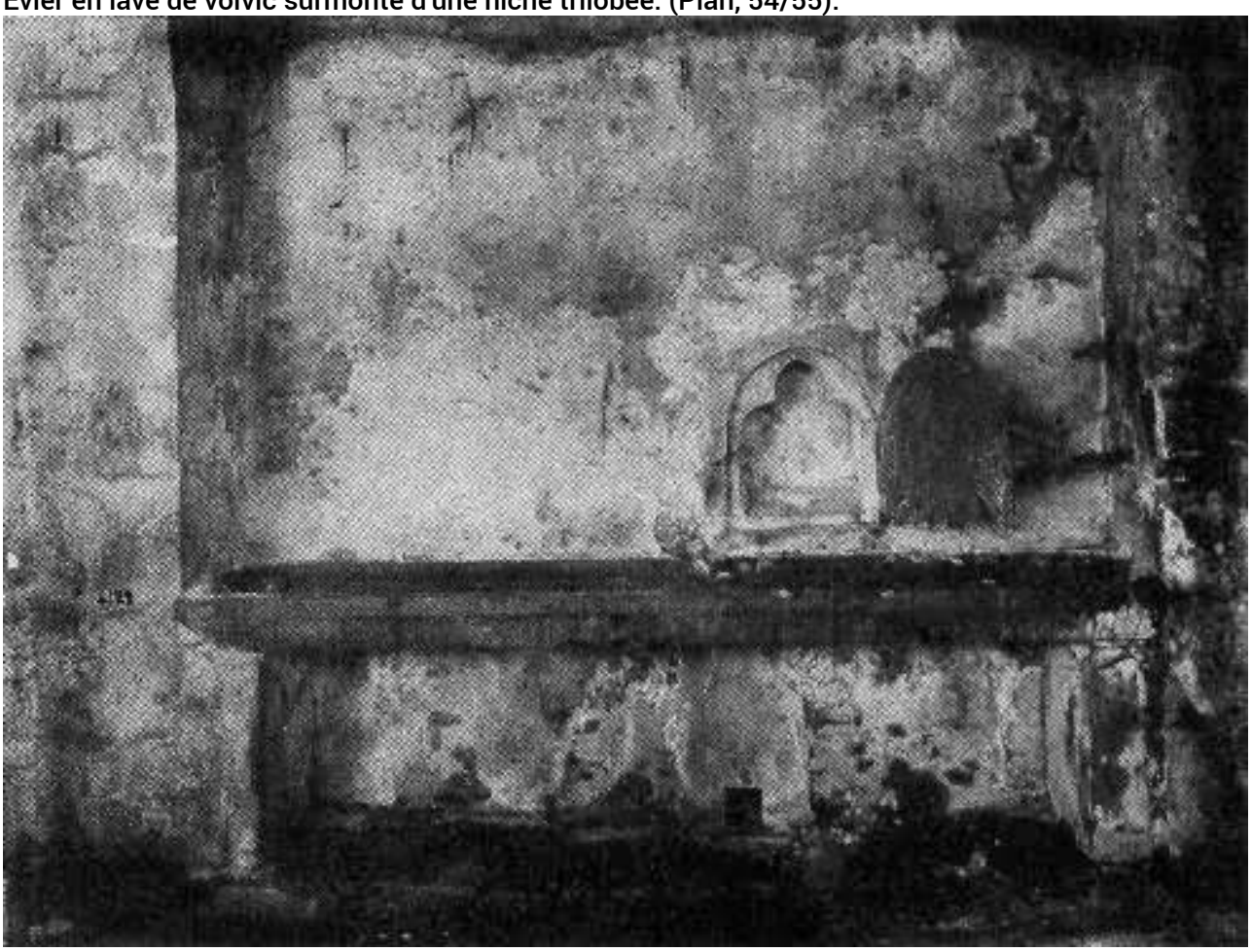

PI. 3

Manufacture française des pneumatiques Michelin, 1912. Tous droits réservés. 


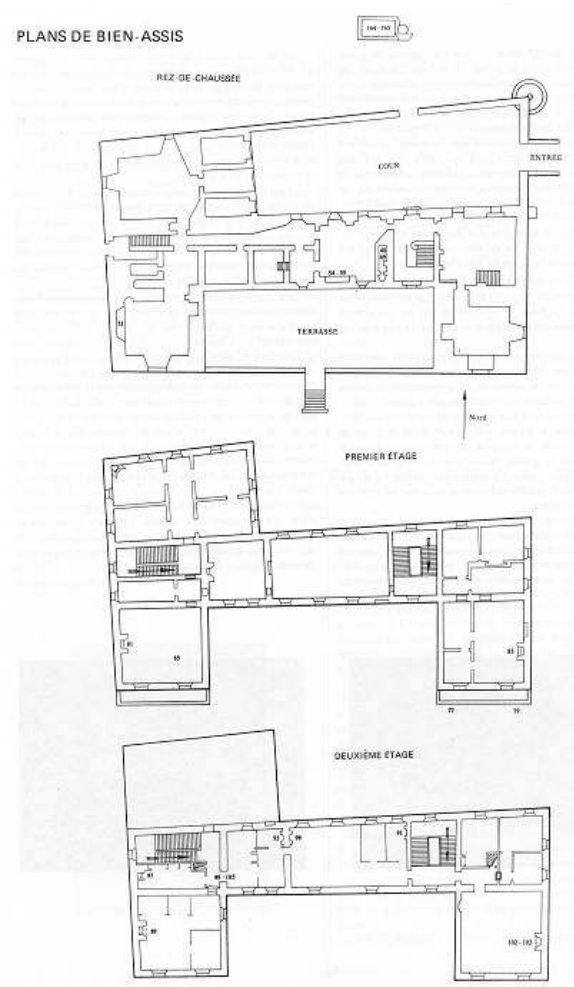

Ces plans ont été dressés par Maurice Marandet, pour l'ouvrage Sur les pas de Blaise Pascal. Le Château de Bien-Assis retrouvé, par Louis Lafuma et Christiane Marandet, pp. 45, 75, 87. Collection « Auvergne et tous les temps », Éditions Volcans, 57, rue Blatin, Clermont-Ferrand, 1964.

$\mathrm{M}^{\text {lle }}$ Christiane Marandet et les Éditions Volcans nous ont gracieusement accordé l'autorisation de les reproduire.

En observant attentivement ces trois plans, on découvre les différentes étapes de la construction de Bien-Assis. En effet l'épaisseur des murs et le style des cheminées du rez-de-chaussée, prouvent que ce niveau est très antérieur ( $\mathrm{XV}^{\mathrm{e}}$ siècle) aux deux étages supérieurs (XVII ${ }^{\mathrm{e}}$ et XVIII ${ }^{\mathrm{e}}$ siècles).

Toutes les photographies accompagnant cet article, à l'exception de celle de la balustrade, sont des clichés de la Manufacture française des pneumatiques Michelin, pris vers 1912. Tous droits réservés.

Les notices sont de Philippe Durin.

(1) Rez-de chaussée : 48-49 : Cheminée ornée d'un écu. - 51 : Cheminée du XVe siècle. - 54-55 : Évier en lave de Volvic - 164-165 : Fontaine et lavoir.

(2) Premier étage : 77 : Façade méridionale du pavillon est. - 79 : Balcon méridional du pavillon est. - 81 : Cheminée pavillon sud-ouest. - 83 : Cheminée pavillon sud-est. -85 : Salon pavillon sud-ouest. 
(3) Deuxième étage : 89 : Cheminée pavillon sud-ouest. - 91 : Cheminée corps de logis central côté nord. - 93 : Cheminée corps de logis central côté nord. - 95 : Porte sur palier pavillon nord-ouest.

- 97 : Cheminée pavillon nord-ouest. - 99 : Cheminée corps de logis central, côté nord. - 102-103 : Cheminée pavillon sud-est. -167 : Lucarnes pavillon sud-est. - 169 :

Lucarnes pavillon, sud-ouest.

Cheminée du XVII e siècle (2 étage). (Plan, 91).

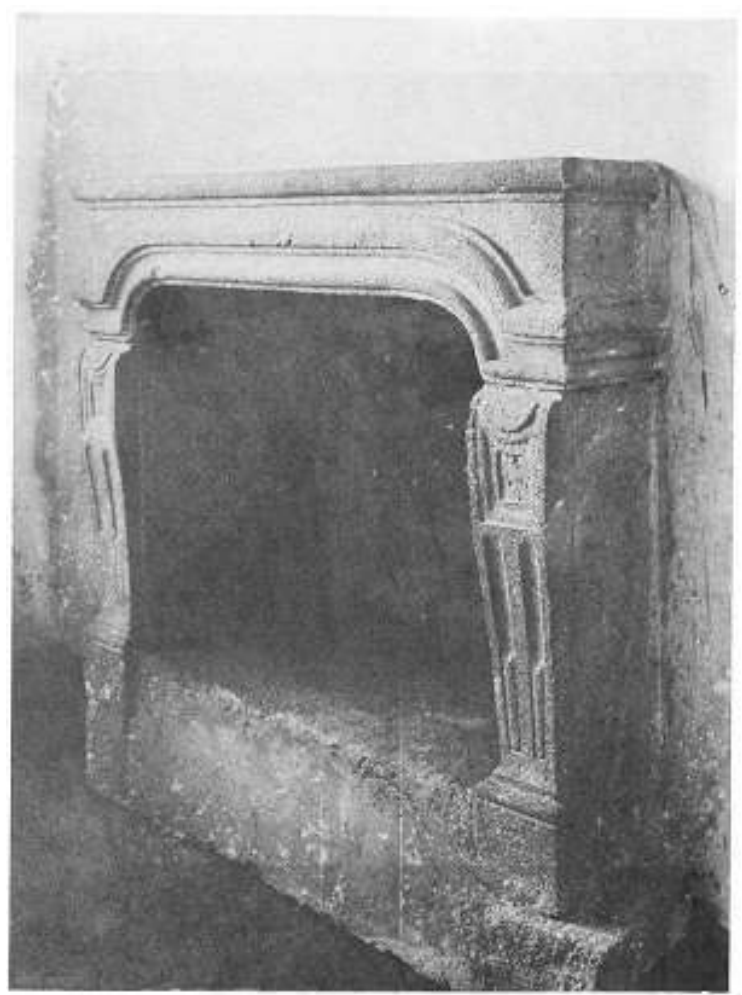

PI. 4 


\section{Cheminée à l'écu}

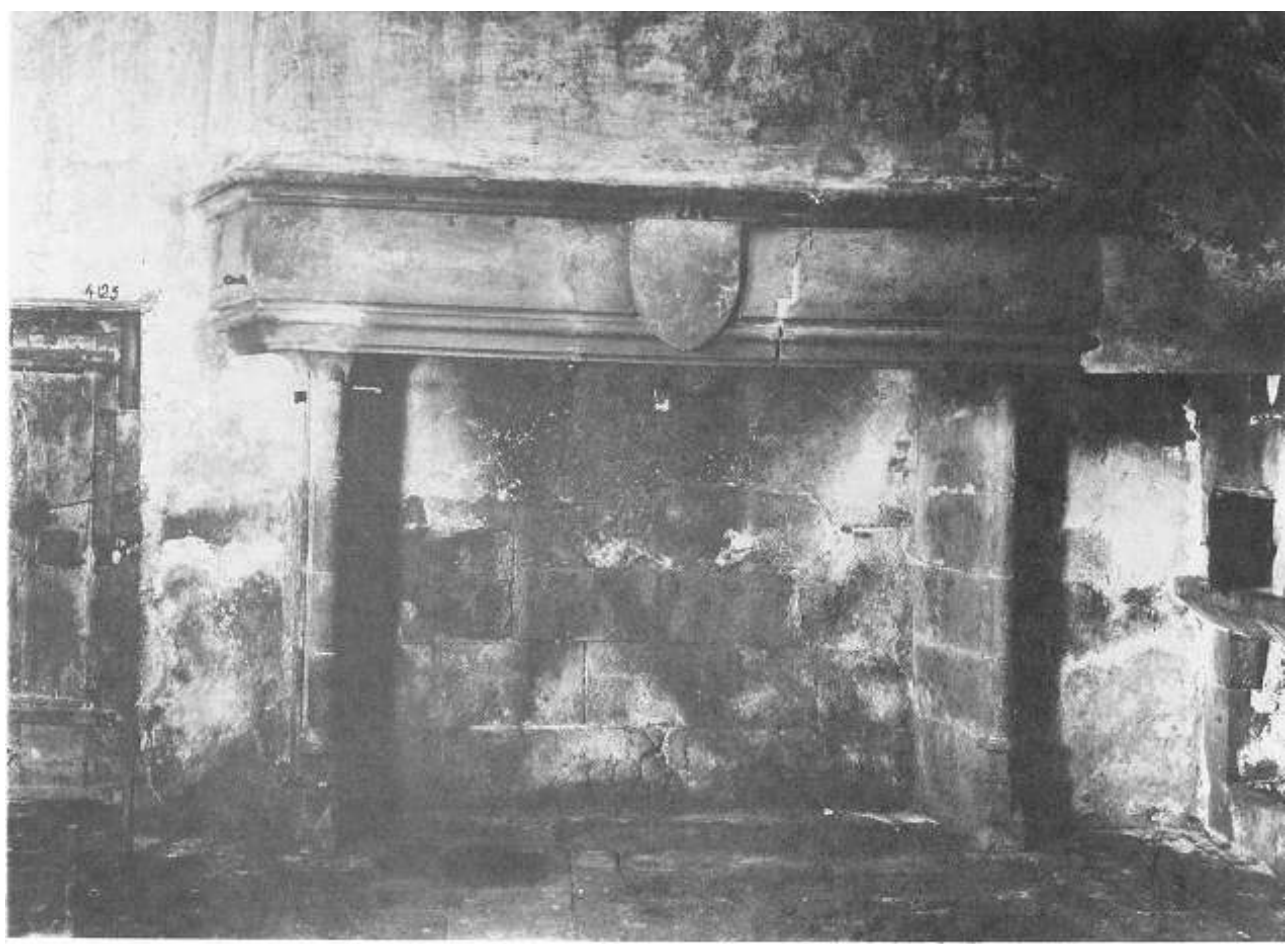

PI. 5. Cette magnifique cheminée monumentale était le principal élément décoratif et utilitaire de la cuisine de Bien-Assis. Elle a été sauvegardée, soigneusement démontée et réhabilitée. De nos jours elle honore un manoir du XVe siècle, aux portes de Clermont, où elle a retrouvé un cadre digne de sa qualité. (Plan, 48/49). 


\section{Cheminée du cabinet des livres}

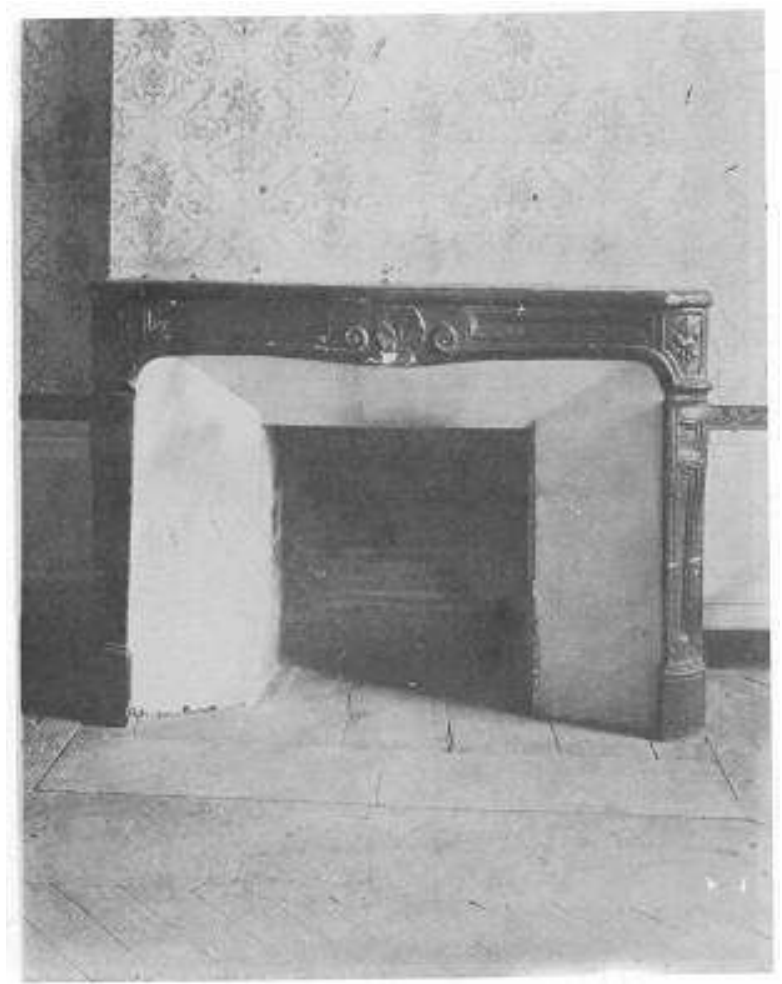

PI. 6 : Le Cabinet des livres est la pièce du château qui a laissé le souvenir le plus émouvant. Son nom est déjà lui-même évocateur de sa vocation réelle. C'était probablement plus un cabinet de travail qu'une bibliothèque d'apparat. On peut croire que Blaise Pascal y a .passé la majeure partie de son temps lors des deux séjours qu'il fit à Bien-Assis. (Plan, 102/103). 


\section{Grande balustrade de la façade Sud}

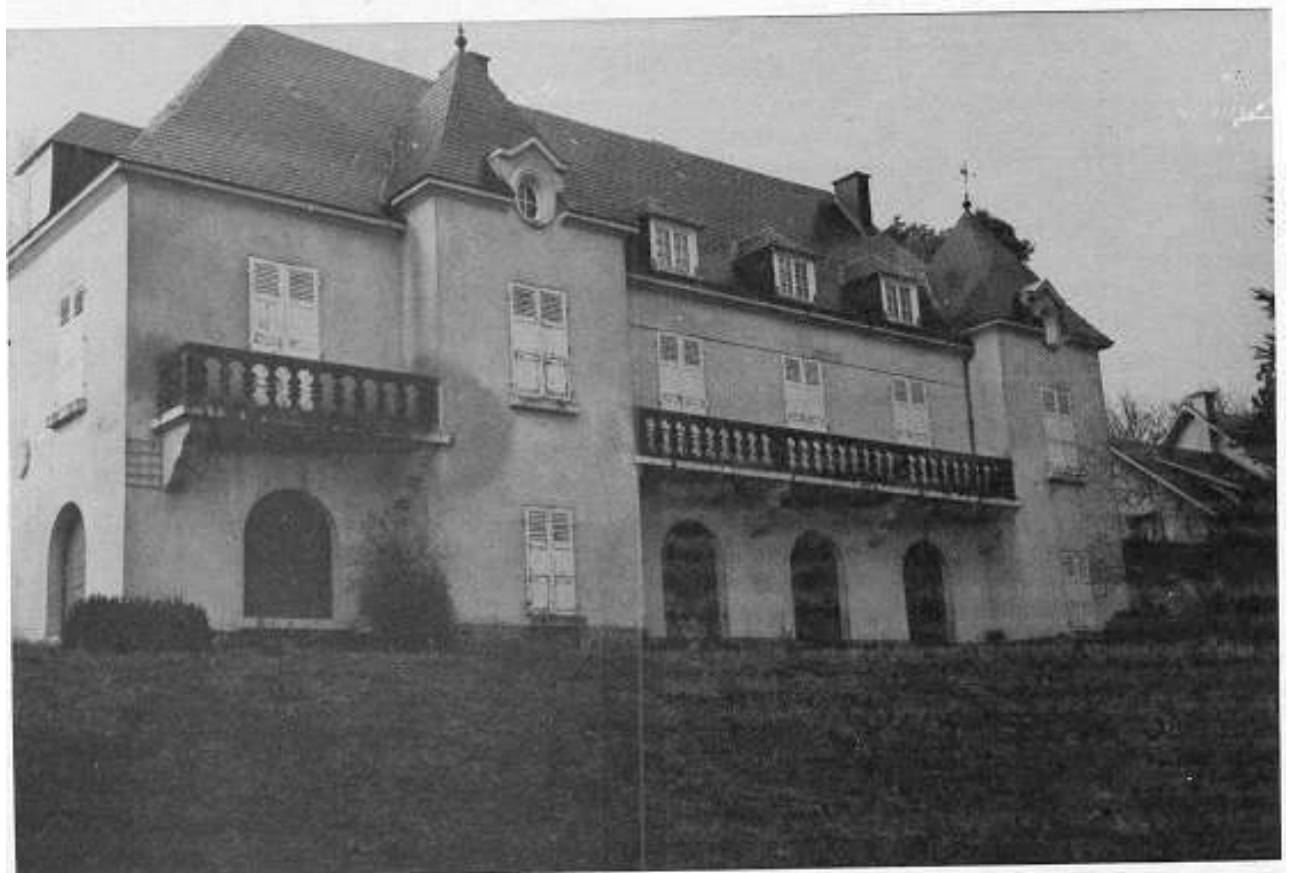

PI. 7 : Les deux corps de logis du château étaient dotés d'une longue balustrade en pierre de Volvic. Lors de la destruction du manoir elle a été sauvée. Maintenant elle orne la façade principale d'une grande villa située au bord du Gour de Tazenat.

\section{Entrée du domaine de Bien-Assis}

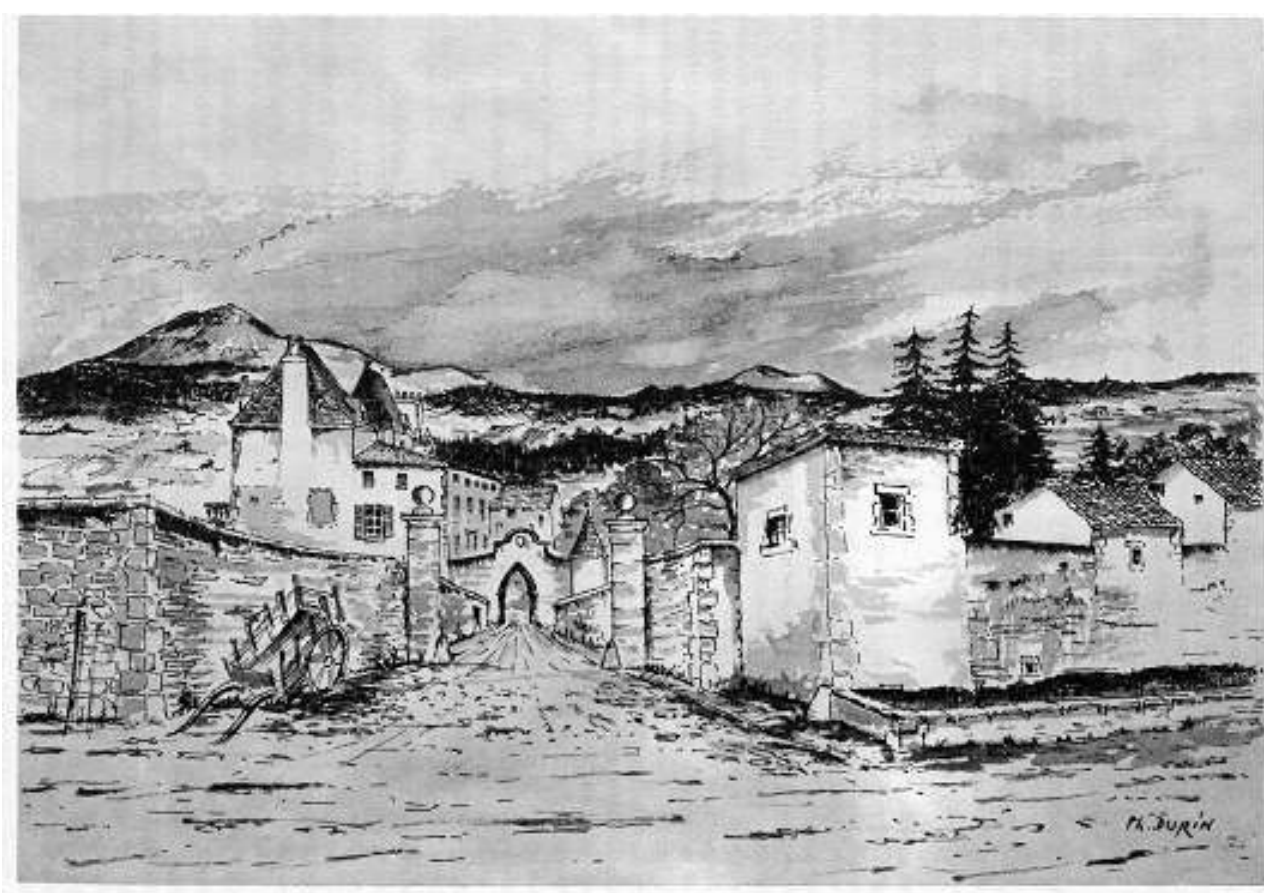

Entrée du domaine de Bien-Assis par le chemin vicinal ordinaire dit « de Bien-Assis ». Reconstitution d'après des plans de la Manufacture française des pneumatiques Michelin. Dessin de Philippe Durin. 